\title{
Defect studies of optical materials using near-field scanning optical microscopy and spectroscopy
}

M. Yan, J. McWhirter, T. Huser, W. Siekhaus

\section{January, 2001}

Lawrence Livermore

National

Laboratory 


\section{DISCLAIMER}

This document was prepared as an account of work sponsored by an agency of the United States Government. Neither the United States Government nor the University of California nor any of their employees, makes any warranty, express or implied, or assumes any legal liability or responsibility for the accuracy, completeness, or usefulness of any information, apparatus, product, or process disclosed, or represents that its use would not infringe privately owned rights. Reference herein to any specific commercial product, process, or service by trade name, trademark, manufacturer, or otherwise, does not necessarily constitute or imply its endorsement, recommendation, or favoring by the United States Government or the University of California. The views and opinions of authors expressed herein do not necessarily state or reflect those of the United States Government or the University of California, and shall not be used for advertising or product endorsement purposes.

Work performed under the auspices of the U. S. Department of Energy by the University of California Lawrence Livermore National Laboratory under Contract W-7405-Eng-48. 
Final Report for LDRD Project - 97-ERD-013

\section{Defect studies of optical materials using near-field scanning optical microscopy and spectroscopy \\ M. Yan, J. McWhirter, T. Huser and W. Siekhaus \\ 97-ERD-013}

Defects and impurities are generally the key material properties that govern the quality and useful lifetime of optical components. Progress in materials synthesis has advanced so that today, for high power laser applications such as the National Ignition Facility (NIF), laser induced damage is typically initiated at the site of sub-micron defects. For example, in laser glass and non-linear optical crystals such as KDP, bulk damage occurs when sub-micron metal inclusion particles absorb laser energy, leading to local heating and eventually material breakdown. It is clear that continued progress in optical materials synthesis is inextricably connected with the need for a detailed understanding of the morphology and composition of the sub-micron damage nucleation sites and the associated damage mechanisms. In this project, we have developed near-field scanning optical microscopy (NSOM) as a tool to optically characterize materials at a spatial resolution of about one order of magnitude better than conventional optical microscopy. Optical microscopy was the first and thus best understood microscopy, and a large number of contrast and spectroscopy techniques have been developed over the years. NSOM is the logical continuation of optical microscopy to the nanometer scale. NSOM combines scanning probe and optical fiber technologies, and simultaneously provides optical (e.g., spectroscopic properties) and topographic information with typically about $50 \mathrm{~nm}$ resolution. This project has resulted in the development of two NSOM instruments, which have been used to perform research on the characterization of surface and subsurface defects in optical materials developed for NIF projects, including fused silica lenses and windows, KDP crystals and color-separation gratings. This research was directly addressed to laserinduced damage in optical materials, which is initiated by a local variation in optical properties at the sub-micron scale.

Our work was focused on the detection of the local laser field associated with sub-micron size defects, using evanescent wave NSOM techniques. 25 years ago, N. Bloembergen proposed that defects such as steep wells and pits on the surface of optical elements produced during polishing cause strong local field-enhancement. If elements with such defects are being used in high-power laser systems they can lead to local dielectric breakdown and consequently laser-induced damage. In NSOM, the detection of the local laser field is achieved by measuring the near-field evanescent wave of a totally internally reflected laser beam at the sample surface. The near-field evanescent wave signal, as collected by NSOM fiber probes, is proportional to the intensity of the electrical field at the sample surface. Figure 1 shows pairs of NSOM images for samples of fused silica, a grating and a KDP crystal. In the figure, the top image of each pair shows the surface topography, and the bottom image is the evanescent wave NSOM image. Images (a) show the topography and local laser intensity of surface damage sites on fused silica. The high intensity in the NSOM image is associated with a surface crack generated during laser irradiation. Subsequent in-situ laser damage was observed to be spatially correlated with the high local laser intensity observed by NSOM. This demonstrates that the local field-enhancement mechanism is important for predicting the growth of laser-induced damage on fused silica. Images (b) show the local field enhancement for an optical 
grating from the LLNL diffractive optics group. In the NSOM image, the high intensity regions are associated with the edge of the grating line structure, as seen in the topographic image. This measurement provides a fundamental understanding for the cause of laser damage and a quantitative analysis of field-enhancement associated with grating structures. Images (c) show etch pits on a KDP crystal surface after coating with a $70 \mathrm{~nm}$ sol-gel anti-reflection coating. The left image shows the flat surface of the sol-gel coating. The right image shows the optical image of the etch pits, which occur at the interface between the sol-gel coating and the KDP crystal. Such etch pits are significant scattering sites and have to be avoided in high-power laser applications. NSOM provides us with a tool to study the initiation and growth of such sub-surface pits on the nanometer scale and will help us to develop recipes on how to avoid them.

These results show that NSOM provides a unique non-destructive method for sub-surface detection of defects. During the final phase of this project we also developed an integrated NSOM/confocal microscopy system with the sensitivity to detect fluorescence from single molecules and to study their properties spectroscopically. This capability opens new avenues for detecting chemical and biological species at the molecular levels.

In summary, we have developed a new metrology to detect defects in optical materials with a resolution that surpasses the diffraction limit of light. We have successfully measured the growth of laser-induced damage in fused silica due to local field-enhancement, local laser intensity variations at the structures of optical gratings and identified etch pits in KDP crystals caused by sol-gel coatings. Thus, NSOM technology provides an optical method to identify and characterize nano-scale materials properties with high significance for both scientific research and programmatic developments at LLNL.
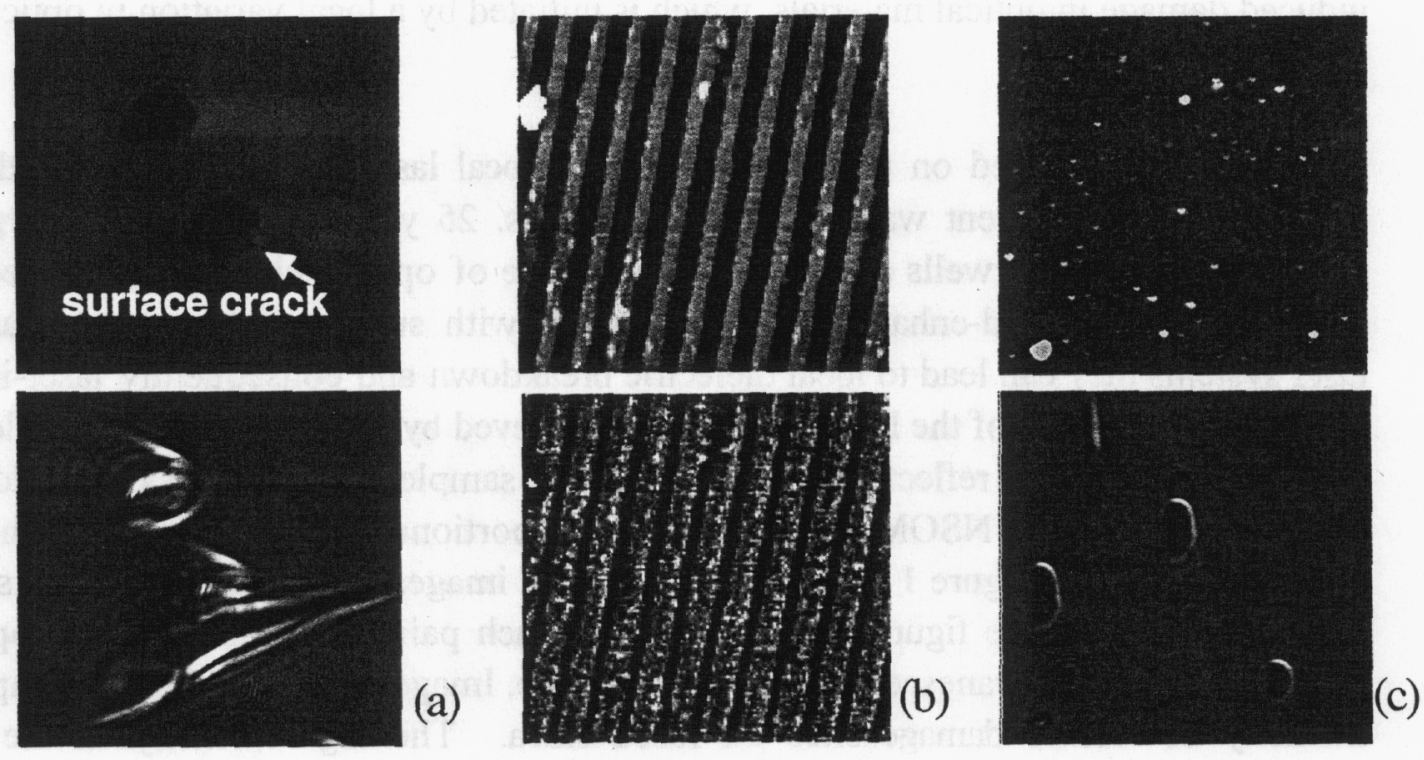

Figure 1. Topography (top) and evanescent-wave NSOM (bottom) images of

(a) Polishing defects on a fused-silica surface, (b) optical gratings and (c) etch pits in sol-gel coated KDP crystal. Image sizes are $20 \mu \mathrm{m}$ x $20 \mu \mathrm{m}$, respectively. 
Final Report for LDRD Project - 97-ERD-013

Publications:

[1] Wang, L., Yan, M., Siekhaus, W., Oberhelman, S.

Probing nanodefects in fused silica by near-field scanning optical microscopy J. Appl. Phys. 84, 3016(1998), UCRL-JC-130635

[2] Yan, M., Wang, L., Siekhaus, W., Kozlowski, M., Yang, J., Mohideen, U. "Defect studies in fused silica using near-field scanning optical microscopy" SPIE Proc. 3244, 268(1997), UCRL-JC-129506 
